\title{
A Low Complexity Near-Optimal MIMO Antenna Subset Selection Algorithm for Capacity Maximisation
}

\author{
Ayyem Pillai Vasudevan ${ }^{1}$ and R. Sudhakar ${ }^{2}$ \\ ${ }^{1}$ Department of ECE, JCT College of Engineering and Technology, Coimbatore 641105, India \\ ${ }^{2}$ Department of ECE, Dr. Mahalingam College of Engineering and Technology, Pollachi 642003, India \\ Correspondence should be addressed to Ayyem Pillai Vasudevan; ayyempillai@yahoo.com
}

Received 26 May 2013; Revised 13 September 2013; Accepted 16 September 2013

Academic Editor: Christoph F. Mecklenbräuker

Copyright (c) 2013 A. P. Vasudevan and R. Sudhakar. This is an open access article distributed under the Creative Commons Attribution License, which permits unrestricted use, distribution, and reproduction in any medium, provided the original work is properly cited.

\begin{abstract}
Multiple input multiple output (MIMO) wireless systems employ a scheme called antenna subset selection for maximising the data rate or reliability for the prevailing channel conditions with the available or affordable number of radio frequency (RF) chains. In this paper, a low-complexity, and near-optimal performance fast algorithm is formulated and the detailed algorithm statements are stated with the exact complexity involved for capacity-maximising receive-only selection. The complexities of other receiveonly selection comparable algorithms are calculated. Complexities have been stated in terms of both complex-complex flops and real-real flops. Significantly, all the algorithms are seen in the perspective of linear increase of capacity with the number of selected antennas up to one less than the total number of receive antennas. It is shown that our algorithm will be a good choice in terms of both performance and complexity for systems, which look for linear increase in capacity with the number of selected antennas up to one less than the total receive antennas. Our algorithm complexity is much less dependent on the number of transmit antennas and is not dependent on the number of selected antennas and it strikes a good tradeoff between performance and speed, which is very important for practical implementations.
\end{abstract}

\section{Introduction}

Multiple input and multiple output (MIMO) wireless systems can be used for increasing Shannon capacity, or decreasing bit error rate through, respectively, spatial multiplexing or diversity. The more the number of antennas, the more will be the capacity and diversity order. But, regardless of spatial multiplexing or diversity concepts, important difficulty in using a MIMO system is an increased complexity and hence cost due to the need of increased radio frequency (RF) chains, which consist of power amplifiers, low noise amplifiers, downconverters, upconverters, and so forth.

This paper focuses on maximising the capacity. Because of the high cost burden involved in RF chains, it is necessary to have less number of RF chains, yet maximise the capacity. This is done by having a larger number of space links at our disposal and selecting the best as many number of links as equal to the number of the RF chains. Selecting the best subset links out of a larger number of links is obviously done by having a larger number of antennas and selecting the best subset of antennas corresponding to the best links. The antenna subset selection can be at the transmit side or at the receive side or at both sides. This paper is concentrating on the selection at the receive side. For a system, which has $M_{R}$ total receive antennas and $M_{T}$ total transmit antennas, the optimal way to select a subset of $L_{R}$ antennas for maximizing capacity is to carry out determinant calculation $\left[\begin{array}{c}M_{R} \\ L_{R}\end{array}\right]$ times as required by the capacity formula given by Telatar [1] and then arrive at the highest capacity-giving antenna subset. Such an exhaustive search method was used in [2] for diversity reception. Similar argument is applicable for transmit side also. Surely, $\left[\begin{array}{c}M_{R} \\ L_{R}\end{array}\right]$ computations of determinants will become prohibitively large. To solve this complexity problem with minimal loss on the capacity performance, suboptimal algorithms have been developed.

Various capacity-based single-sided antenna selection problems have been discussed in the literature [3-13]. In [3], 
antenna selection was considered at the transmitter for a low rank channel. In this paper, there was no mention of RF chain constraint concept with regard to antenna selection; rather, an optimization criterion was built up for maximizing the capacity and the algorithm of antenna selection was left as a future direction. In [5], an SNR-based criterion was developed for antenna selection in transmitter side for spatial multiplexing systems employing zero forcing-based linear receivers. In [6], the concept of antenna selection with respect to the RF chain was introduced by Molisch et al. In that paper, an analytical bound for the capacity was derived. The system was called hybrid selection MIMO (H-S/MIMO), where in the case of standard diversity, it is called as hybrid selection maximum ratio combining (H-S/MRC). In [7], a selection rule for maximizing the average throughput was given for transmit antenna selection of spatial multiplexing systems. A norm-based antenna selection algorithm for transmit side was suggested by Gore et al. for the Alamouti space time coding system in [8]. In this, antennas of largest Euclidean norms were selected, where it is to be noted that the normbased algorithm can be very suboptimaly. Norm-based selection can also be used for capacity criterion as suggested in [9]. A high-capacity achieving suboptimal antenna selection algorithm, whose performance in terms of capacity, is very close to that of the optimal one, and whose computational complexity is not that promising, was given by Gorokhov in [10]. This algorithm, which comes under greedy search, started with the full set of antennas and one by one deleted the least capacity contributing antenna. This algorithm also directly dealt with Telatar's exact capacity expression [1]. This expression is shown as (1) later. The algorithm changed the problem of finding determinant into finding an inverse for the purpose of reducing the computational complexity. However, the inverse lemma used in the algorithm demands a huge number of flops. The complexity of this algorithm was calculated to be $O\left(M_{T}^{3}+M_{T}^{2} M_{R}\right)+O\left(M_{R}^{2} M_{T}^{2}\right)+O\left(M_{R}^{2}\right)+$ $O\left(M_{T}^{2} M_{R}\right)+O\left(M_{T}^{2} M_{R}\right)$ in [11] for receive side selection. It can be noted that for receive side selection, it involves not only the total number of receive side antennas but also the total transmit antennas. Hence, this algorithm is computationally complex. A follow-up to [10] was made in [11] by Gharavi-Alkhansari and Gershman, who introduced an addition-based greedy sub-optimal algorithm for receive side selection. In that paper, the authors followed the procedure of starting with empty set of antennas and then added one by one maximum capacity contributing antenna. They used the well-known capacity equation given as (1) later, for their algorithm. The authors of [11] also changed the problem of finding determinants to finding inverse as done in [10], but the difference is that computation for finding the inverse was dramatically reduced by using a lemma that finds the inverse by addition and matrix multiplication. A computationally and constructionally simple algorithm was given by Molisch et al. in [12]. This algorithm does not directly deal with (1) and comes under the classification of fast selection sub-optimal algorithms group, where, it is understood that this kind of algorithms does not use directly (1) and surely there will be a small compromise on capacity. Clearly, this algorithm is simple, but there is considerable penalty in terms of capacity. In this, correlation between each pair of the rows, where rows correspond to receive antennas, is found and sequentially the first $M_{R}-L_{R}$ highest correlation antennas are deleted for selecting $L_{R}$ antennas. After getting a particular high correlation antenna pair, the antenna corresponding to the lower norm row is deleted. In [13], an antenna selection algorithm based on Tanimoto similarity was proposed. However, this algorithm is computationally complex as it involves several matrix multiplications. Rate adaptation and single transmit antenna selection were studied in [4].

Various articles have been published on the antenna selection at both sides for capacity maximisation. The paper have been on decoupled transmit/receive $(\mathrm{Tx} / \mathrm{Rx})$ antenna selection and greedy joint selection. The concept of both side selection was first proposed in [14]. The authors suggested decoupling or separating selections at transmit and receive sides. They suggested optimal search separately on receive antenna and transmit antenna sides reducing the otherwise required calculation of $\left[\begin{array}{c}M_{R} \\ L_{R}\end{array}\right]\left[\begin{array}{c}M_{T} \\ L_{T}\end{array}\right]$ determinants to calculation of $\left[\begin{array}{c}M_{R} \\ L_{R}\end{array}\right]+\left[\begin{array}{c}M_{R} \\ L_{R}\end{array}\right]$ determinants without affecting the capacity much. Further in [9], the decoupling concept was suggested in terms of greedy algorithm of [11] at both sides. A greedy joint algorithm called efficient joint transmit and receive antenna selection (EJTRAS) algorithm was proposed for capacity maximisation in [15].

Gharavi-Gershman's receive-side greedy algorithm achieves good capacity performance, but its complexity is clearly a function of the number of transmitting antennas and the number of selected antennas. Their algorithm can be claimed to be of low complexity only for the case of low transmitting antenna number case. The complexity is of the order of $M_{T}^{2}$, whereas reduction in $M_{T}$ will surely impose constraint on increase in $L_{R}$, because if $L_{R}>M_{T}$, linear increase in capacity is not possible. These issues are brought out quantitatively in Sections 4 and 5. Though the algorithm proposed in [12] is devoid of these constraints, the algorithm is considerably sub-optimal.

In this paper, the authors propose for receive side an addition-based fast algorithm, which is considerably superior in performance to algorithm given in [12] with only very small percentage increase in complexity as the simulations and computation calculations show. Our algorithm achieves a capacity, that is, almost equal to greedy algorithm proposed in [11] but, it is computationally independent of number of selected antennas and very importantly, its complexity is much less dependent on the number of transmitting antennas unlike the one in [11]. Our algorithm is devoid of the constraint imposed by greedy algorithm of [11] and is superior in performance to that in [12].

The paper has been organized as follows. Section 2 gives the theoretical model for capacity-based antenna selection at the receive side. In Section 3, the algorithm is developed, so that it takes better care of norm than that in [12] and the proposed algorithm and its pseudostatements with complexity involved are stated. In Section 4, the computational complexities of all the above mentioned algorithms are discussed with comparison. Section 5 is on simulation considerations, 


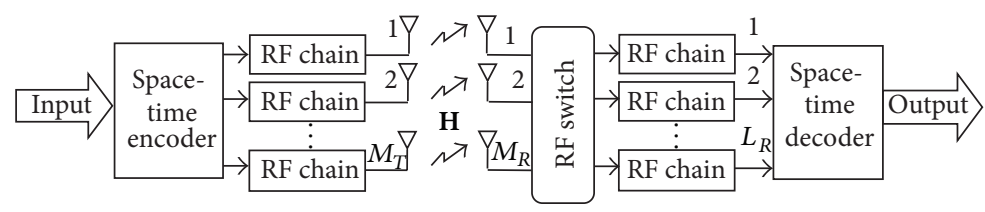

FIGURE 1: A MIMO system with receive side antenna subset selection.

results and discussions. Section 6 gives conclusion. Table 5 gives the complexity break-up of Gharavi et al. algorithm. Table 6 gives the complexity break-up of Molisch et al. algorithm.

Notations. All the bold and lower case letters refer to matrix vectors, whereas all the bold and upper case letters refer to matrices. Superscript $H$ refers to Hermitian transpose. The following definitions are applicable for the whole paper.

$\mathbf{H} \triangleq$ Channel matrix of $M_{R} \times M_{T}$.

$M_{R} \triangleq$ Number of available receive antennas.

$M_{T} \triangleq$ Number of available transmitting antennas.

$\rho \triangleq$ Symbol energy to noise power ratio.

$\mathbf{I}_{M_{R}} \triangleq$ Unit matrix of $M_{R}$ dimension.

$\mathbf{H}_{p,:} \triangleq p$ th row vector of $\mathbf{H}$.

$\mathfrak{E}_{R} \triangleq$ The selected antenna subset.

$L_{R} \triangleq$ The number of selected antennas.

$\mathbf{H}_{\mathfrak{E}_{R} \text {; }} \triangleq$ The sub-channel matrix formed of rows corresponding to selected antennas and all columns of $\mathbf{H}$.

$\lambda_{i} \triangleq$ Eigen values of $\mathbf{H H}^{H}$.

$\theta \triangleq$ Angle between two row vectors.

$\left\langle\mathbf{h}_{i}, \mathbf{h}_{j}\right\rangle \triangleq$ Inner product between vectors $\mathbf{h}_{i}$ and $\mathbf{h}_{j}$.

$C \triangleq$ Channel capacity of MIMO system.

$\left\|\mathbf{h}_{i}\right\| \triangleq$ Euclidean norm of vector $\mathbf{h}_{i}$.

\section{The System Model}

The capacity of a MIMO system that uses $M_{R}$ receiving antennas and $M_{T}$ transmitting antennas fed of equal power is given by

$$
C=\log _{2} \operatorname{det}\left[\mathbf{I}_{M_{R}}+\frac{\rho}{M_{T}} \mathbf{H H}^{H}\right] .
$$

This expression can be brought to (2) by using singular value decomposition (SVD). Consider

$$
C=\sum_{i=1}^{r} \log \left[\frac{\rho}{M_{T}} \lambda_{i}+1\right] \mathrm{bps} / \mathrm{Hz},
$$

where $r$ is the number of nonzero eigen values. Hence, it suggests that $C$ depends on not only the number of independent columns or the number of independent rows but also on eigen value distribution. Simple independence is only a weak requirement as far as capacity is concerned. Capacity can be actually maximized by having not just independent columns or rows but by having orthogonal columns or rows. That is, the eigen values of orthogonal columns or rows will ensure that the capacity is maximized.

Antenna selection is an approach, which tries to extract maximum benefit out of the prevailing channel conditions with the number of available or affordable RF chains. If available RF chains are only $L_{R}$, effective receive antennas can be only $L_{R}$ in number. Since only $L_{R}$ antennas are to be selected, (1) will change to

$$
C_{\text {select }}=\log _{2} \operatorname{det}\left[\mathbf{I}_{L_{R}}+\frac{\rho}{M_{T}} \mathbf{H}_{\mathfrak{E}_{R},:} \mathbf{H}_{\mathfrak{E}_{R},:}^{\mathbf{H}}\right] \text {. }
$$

The MIMO system with antenna subset selection approach for receive side is depicted in Figure 1.

\section{The Proposed Algorithm}

In this section, a new uncorrelation-based algorithm is logically brought for receiver side selection. The expression for the capacity with $L_{R}$ receive antennas can be written with the application of SVD on it as given in (1).

In [12], Molisch et al. proposed an algorithm, based on correlation among the rows, for receive side antenna subset selection. The idea was to remove the correlated rows and retain as far as possible maximally uncorrelated or optimally orthogonal rows. Molisch et al. algorithm is simple and of low complexity, but the capacity performance is considerably suboptimal.

The reason for the underperformance of Molisch et al. algorithm is that one of the two rows, which have high correlation between them and high individual norm values will get deleted. That is, the norm values are less accounted in that algorithm. But norm values matter as suggested by product $\mathbf{H H}^{H}$ in (1). The correlation or nonorthogonality between two rows is measured in terms of the inner product $\mathbf{h}_{i} \mathbf{h}_{j}^{H}$. The existence of high correlation among any two rows will mean that the inner product among the rows is high.

Let us consider two rows, $\mathbf{h}_{i}$ and $\mathbf{h}_{j}$ of $\mathbf{H}$, where

$$
\mathbf{H}=\left[\begin{array}{c}
\mathbf{h}_{1} \\
\mathbf{h}_{\mathbf{2}} \\
\mathbf{h}_{\mathbf{3}} \\
\vdots \\
\mathbf{h}_{M_{R}}
\end{array}\right] .
$$

The rows $\mathbf{h}_{\mathbf{1}}, \mathbf{h}_{\mathbf{2}}, \mathbf{h}_{3}, \ldots, \mathbf{h}_{M_{R}}$ are of $1 \times M_{T}$. The inner product between two rows, $\mathbf{h}_{i}$ and $\mathbf{h}_{j}$, can be written as

$$
\mathbf{h}_{i} \mathbf{h}_{j}^{H}=\left\|\mathbf{h}_{i}\right\|\left\|\mathbf{h}_{j}\right\| \cos \theta .
$$




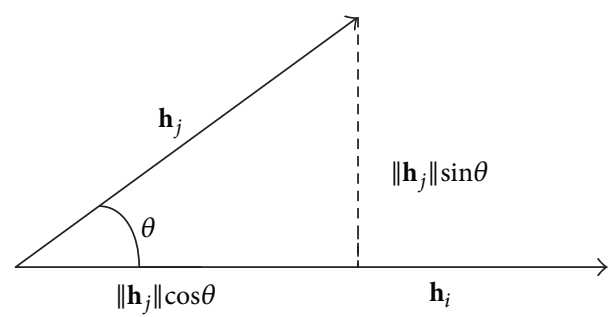

FIGURE 2: Interpretation of inner product between $\mathbf{h}_{j}$ and interpretation of resolution of $\mathbf{h}_{j}$ along $\mathbf{h}_{i}$ and perpendicular to $\mathbf{h}_{i}$.

That is, correlation $=\left\|\mathbf{h}_{i}\right\|\left\|\mathbf{h}_{j}\right\| \cos \theta$, where $i=1,2,3, \ldots, M_{R}$, $j=1,2,3, \ldots, M_{R}$. Figure 2 describes the inner product.

In this, $\left\|\mathbf{h}_{j}\right\| \cos \theta$ is the projection of $\mathbf{h}_{j}$ vector on $\mathbf{h}_{i}$ vector. Hence, correlation is the norm of vector $\mathbf{h}_{i}$ multiplied by the component of $\mathbf{h}_{j}$ along $\mathbf{h}_{i}$. The resolution of $\mathbf{h}_{j}$ is shown in Figure 2 . The uncorrelation between $\mathbf{h}_{i}$ and $\mathbf{h}_{j}$ will be the norm of $\mathbf{h}_{i}$ multiplied by the component perpendicular to $\mathbf{h}_{i}$ where the perpendicular is $\left\|\mathbf{h}_{j}\right\| \sin \theta$. That is, the uncorrelation is given by

$$
\text { uncorrelation }=\left\|\mathbf{h}_{i}\right\|\left\|\mathbf{h}_{j}\right\| \sin \theta .
$$

Let us define

$$
\begin{aligned}
& \alpha_{\mathbf{h}_{i} \mathbf{h}_{j}} \triangleq \text { correlation between } \mathbf{h}_{i} \text { and } \mathbf{h}_{j} \\
& \beta_{\mathbf{h}_{i} \mathbf{h}_{j}} \triangleq \text { uncorrelation between } \mathbf{h}_{i} \text { and } \mathbf{h}_{j} .
\end{aligned}
$$

Then,

$$
\begin{aligned}
\alpha_{\mathbf{h}_{i} \mathbf{h}_{j}}^{2}+\beta_{\mathbf{h}_{i} \mathbf{h}_{j}}^{2} & =\left\|\mathbf{h}_{i}\right\|^{2}\left\|\mathbf{h}_{j}\right\|^{2} \cos ^{2} \theta+\left\|\mathbf{h}_{i}\right\|^{2}\left\|\mathbf{h}_{j}\right\|^{2} \sin ^{2} \theta \\
& =\left\|\mathbf{h}_{i}\right\|^{2}\left\|\mathbf{h}_{j}\right\|^{2}\left[\cos ^{2} \theta+\sin ^{2} \theta\right] \\
& =\left\|\mathbf{h}_{i}\right\|^{2}\left\|\mathbf{h}_{j}\right\|^{2} .
\end{aligned}
$$

Hence, the square of uncorrelation is given by

$$
\beta_{\mathbf{h}_{i} \mathbf{h}_{j}}^{2}=\left\|\mathbf{h}_{i}\right\|^{2}\left\|\mathbf{h}_{j}\right\|^{2}-\alpha_{\mathbf{h}_{i} \mathbf{h}_{j}}^{2}=\left\|\mathbf{h}_{i}\right\|^{2}\left\|\mathbf{h}_{j}\right\|^{2}-\left(\mathbf{h}_{i} \mathbf{h}_{j}^{H}\right)^{2} .
$$

Molisch et al. algorithm was about finding the correlation and deleting the rows corresponding to high correlation, where correlation between two vectors was defined to be the inner product between the two row vectors. But the problem is that high inner products may be due to high values of norms of the vectors and not necessarily due to high values of $\cos \theta$ alone, in which case deletion of any one vector will penalize the capacity of the system as the product $\mathbf{H H}^{H}$ suggests in (1). But, if the same algorithm is steered to addition concept, such a scenario of deletion of highnorm rows will not happen. Because in addition concept, the uncorrelation between the rows is found and those rows corresponding to high values of uncorrelation are retained. Hence, high norm rows will be retained rather than deleted.

We have uncorrelation given by

$$
\beta_{\mathbf{h}_{i} \mathbf{h}_{j}}^{2}=\left\|\mathbf{h}_{i}\right\|^{2}\left\|\mathbf{h}_{j}\right\|^{2}-\left\|\mathbf{h}_{i}\right\|^{2}\left\|\mathbf{h}_{j}\right\|^{2} \cos ^{2} \theta \text {. }
$$

In the following, it will be seen that Molisch et al. algorithm does care about correlation aspect well but does not give due care for norm. For this, we take two pairs of rows, in which pair, 1 is more correlated than pair 2 by some percent, but the norm product of pair 1 is more than pair 2 by more percent. Let us consider $(i, j)$ pair of rows and $(k, l)$ pair of rows. Let $\theta_{1}$ be between $i$ th and $j$ th rows and let $\theta_{2}$ be between $k$ th and $l$ th rows. Let square of $\left\langle\mathbf{h}_{i}, \mathbf{h}_{j}\right\rangle$ be 1 percent greater than square of $\left\langle\mathbf{h}_{k}, \mathbf{h}_{l}\right\rangle$ and let $\left\|\mathbf{h}_{i}\right\|^{2}\left\|\mathbf{h}_{j}\right\|^{2}$ be 2 percent greater than $\left\|\mathbf{h}_{k}\right\|^{2}\left\|\mathbf{h}_{l}\right\|^{2}$. This means that the correlation between $\mathbf{h}_{i}$ and $\mathbf{h}_{j}$ is 1 percent greater than the correlation between $\mathbf{h}_{k}$ and $\mathbf{h}_{l}$. Hence, $\mathbf{h}_{i}$ and $\mathbf{h}_{j}$ are more correlated than $\mathbf{h}_{k}$ and $\mathbf{h}_{l}$. We will assume that each of $\left\|\mathbf{h}_{i}\right\|^{2}$ and $\left\|\mathbf{h}_{j}\right\|^{2}$ is greater than both $\left\|\mathbf{h}_{k}\right\|^{2}$ and $\left\|\mathbf{h}_{l}\right\|^{2}$. This is a situation of square norms multiplication of two rows, $i$ and $j$ being greater than square norms multiplication of the other two rows, $k$ and $l$, whereas the $\cos \theta_{1}$ is lower than $\cos \theta_{2}$. Under the abovestated assumptions, the uncorrelation becomes

$$
\begin{aligned}
& \beta_{\mathbf{h}_{i} \mathbf{h}_{j}}^{2}= {\left[\left\|\mathbf{h}_{k}\right\|^{2}\left\|\mathbf{h}_{l}\right\|^{2}+0.02\left\|\mathbf{h}_{k}\right\|^{2}\left\|\mathbf{h}_{l}\right\|^{2}\right] } \\
&-\left\|\mathbf{h}_{k}\right\|^{2}\left\|\mathbf{h}_{l}\right\|^{2} \cos ^{2} \theta_{2}-0.01\left\|\mathbf{h}_{k}\right\|^{2}\left\|\mathbf{h}_{l}\right\|^{2} \cos ^{2} \theta_{2} \\
&= {\left[\left\|\mathbf{h}_{k}\right\|^{2}\left\|\mathbf{h}_{l}\right\|^{2}-\left\|\mathbf{h}_{k}\right\|^{2}\left\|\mathbf{h}_{l}\right\|^{2} \cos ^{2} \theta_{2}\right] } \\
&+0.02\left\|\mathbf{h}_{k}\right\|^{2}\left\|\mathbf{h}_{l}\right\|^{2}-0.01\left\|\mathbf{h}_{k}\right\|^{2}\left\|\mathbf{h}_{l}\right\|^{2} \cos ^{2} \theta_{2} \\
&= \beta_{\mathbf{h}_{k} \mathbf{h}_{l}}^{2}+\left[0.02\left\|\mathbf{h}_{k}\right\|^{2}\left\|\mathbf{h}_{l}\right\|^{2}\right. \\
&\left.-0.01\left\|\mathbf{h}_{k}\right\|^{2}\left\|\mathbf{h}_{l}\right\|^{2} \cos ^{2} \theta_{2}\right] .
\end{aligned}
$$

The bracketed part of (10) will be a +ve value because, $0.02\left\|\mathbf{h}_{k}\right\|^{2}\left\|\mathbf{h}_{l}\right\|^{2}$ will be greater than $0.01\left\|\mathbf{h}_{k}\right\|^{2}\left\|\mathbf{h}_{l}\right\|^{2} \cos ^{2} \theta_{2}$. Hence, we see that $\beta_{\mathbf{h}_{i} \mathbf{h}_{j}}^{2}$ will be greater than $\beta_{\mathbf{h}_{k} \mathbf{h}_{l}}^{2}$. Hence, if our algorithm is used, we can expect that the higher-norm row of $i, j$ rows, whose individual norms are greater than the individual norms of $k, l$ rows, will be selected. Due to this phenomenon, the proposed algorithm will fetch more capacity than that done by Molisch et al. algorithm and we see in the simulation this is the case. If Molisch et al. algorithm is applied, one among $i$ th and $j$ th rows will be deleted because $\left\langle\mathbf{h}_{i}, \mathbf{h}_{j}\right\rangle$ is higher than $\left\langle\mathbf{h}_{k}, \mathbf{h}_{l}\right\rangle$. This will cause a reduction in capacity because, among the rows $i$ and $j$, one may be deleted. The proposed algorithm is stated next, and its statement version with the complexity involved is stated in Table 1. Consider the following.

(1) The channel vector $\mathbf{h}_{i}$ is defined as the $i$ th row of $\mathbf{H}$, with $i$ being an element of the set $S_{R}=\left\{1,2, \ldots, M_{R}\right\}$.

(2) If $L_{R}=M_{R}$, conclude $\mathfrak{E}_{R}=S_{R}$; otherwise, do the steps 3 to 7 .

(3) For all $i$ (with $i \in S_{R}$ ), calculate square norm of $\mathbf{h}_{i},\left\|\mathbf{h}_{i}\right\|^{2}$. The square uncorrelation $\beta_{\mathbf{h}_{i} \mathbf{h}_{j}}^{2}$ is defined as $\beta_{\mathbf{h}_{i} \mathbf{h}_{j}}^{2}=\left\|\mathbf{h}_{i}\right\|^{2}\left\|\mathbf{h}_{j}\right\|^{2}-\alpha_{\mathbf{h}_{i} \mathbf{h}_{j}}^{2}, \alpha_{\mathbf{h}_{i} \mathbf{h}_{j}}^{2}$ being the square correlation. The square correlation $\alpha_{\mathbf{h}_{i} \mathbf{h}_{j}}^{2}$ is defined as $\alpha_{\mathbf{h}_{i} \mathbf{h}_{j}}^{2}=$ square $\left(\left|\left\langle\mathbf{h}_{i}, \mathbf{h}_{j}\right\rangle\right|\right)$. 
TABLE 1: Pseudostatements and complexity details of the proposed algorithm.

\begin{tabular}{|c|c|c|c|}
\hline Sl. no. & Algorithm statements & Complex flops required & Real flops required \\
\hline 1 & $S_{R}=\left\{1,2,3, \ldots, M_{R}\right\}$ & \multirow{5}{*}{$2 M_{R} M_{T}-M_{R}$} & \multirow{5}{*}{$8 M_{R} M_{T}-4 M_{R}$} \\
\hline 2 & if $L_{R}<M_{R}$ & & \\
\hline 3 & for $j=1$ to $M_{R}$ & & \\
\hline 4 & $\gamma_{j}^{2}=\left\|\mathbf{H}_{j,:}\right\|^{2}$ & & \\
\hline 5 & end & & \\
\hline 6 & if $L_{R}>1$ & \multirow{9}{*}{$\frac{\left(2 M_{T}+2\right)\left(M_{R}^{2}-M_{R}\right)}{2}$} & \multirow{9}{*}{$\left(4 M_{T}-0.5\right)\left(M_{R}^{2}-M_{R}\right.$} \\
\hline 7 & for $p=1$ to $M_{R}$ & & \\
\hline 8 & for $q=1$ to $M_{R}$ & & \\
\hline 9 & if $p>q$ & & \\
\hline 10 & $\alpha_{p, q}=\left(\mathbf{H}_{p::} \mathbf{H}_{q,:}^{H}\right)$ & & \\
\hline 11 & $\beta_{p, q}=\left|\gamma_{p}^{2} \gamma_{q}^{2}-\alpha_{p, q}^{2}\right|$ & & \\
\hline 12 & end & & \\
\hline 13 & end & & \\
\hline 14 & end & & \\
\hline 15 & for $n=1$ to $L_{R}$ & & \\
\hline 16 & $P, Q=\underset{p, q \in S_{R}}{\arg \max } \beta_{p, q}$ & & \\
\hline 17 & if $\left\|\mathbf{H}_{P,}\right\|^{2}>\left\|\mathbf{H}_{Q:}\right\|^{2}$ & & \\
\hline 18 & $S_{R}=S_{R}-P$ & & \\
\hline 19 & $\beta_{P,:}=0$ & & \\
\hline 20 & else & & \\
\hline 21 & $S_{R}=S_{R}-Q$ & & \\
\hline 22 & $\beta_{\mathrm{Q}, ;}=0$ & & \\
\hline 23 & end & & \\
\hline 24 & end & & \\
\hline 25 & else & & \\
\hline 26 & $J=\underset{j \in S_{R}}{\arg \max } \gamma_{j}^{2}$ & & \\
\hline 27 & $S_{R}=S_{R}-J$ & & \\
\hline 28 & end & & \\
\hline 29 & $\mathfrak{E}_{R}=\left\{1,2, \ldots, M_{R}\right\}-S_{R}$ & & \\
\hline 30 & end & & \\
\hline
\end{tabular}

(4) If $L_{R}=1$, select $i$ (with $i \in S_{R}$ ), that gives the largest $\left\|\mathbf{h}_{i}\right\|^{2}$, and conclude $\mathfrak{E}_{R}=\{i\}$; otherwise, do steps 5 to 7 .

(5) For all $i$ and $j, i>j$, compute the square correlation, $\alpha_{\mathbf{h}_{i} \mathbf{h}_{j}}^{2}=$ square $\left(\left|\left\langle\mathbf{h}_{i}, \mathbf{h}_{j}\right\rangle\right|\right)$.

(6) For all $i$ and $j, i>j$, compute the square uncorrelation, $\beta_{\mathbf{h}_{i} \mathbf{h}_{j}}^{2}=\left\|\mathbf{h}_{i}\right\|^{2}\left\|\mathbf{h}_{i}\right\|^{2}-\alpha_{\mathbf{h}_{i} \mathbf{h}_{j}}^{2}$.

(7) For Loop, Consider the following.

(a) Choose the $i$ and $j$ (with $i, j \in S_{R}, i>j$ ) that give the largest $\beta_{\mathbf{h}_{i} \mathbf{h}_{j}}^{2}$. If $\left\|\mathbf{h}_{i}\right\|^{2}>\left\|\mathbf{h}_{j}\right\|^{2}$, add $i$ to $\mathfrak{E}_{R}$, otherwise, and add $j$ to $\mathfrak{E}_{R}$.

(b) Delete $i$ (or $j$ ) from $S_{R}$.

(c) Go to Loop until $L_{R}$ indices are in $\mathfrak{E}_{R}$.

\section{Complexity Analysis of the Algorithms}

In this section, a detailed analysis has been made on the complexity of the proposed Molisch et al., and GharaviGershman algorithms. For complexity analysis, the numbers of multiplications and summations have been accounted. The multiplications and summations are together called as floating point operations, abbreviated as flops. The number of flops demanded by each of the three is taken as the benchmark for comparison of complexities of the algorithms. Firstly, complex-complex flops and then real-real flops are calculated. Each complex-complex multiplication involves four real multiplications and two real additions. $N$ complexcomplex additions involve $2 \mathrm{~N}-2$ real-real additions.

In the case of the proposed algorithm, for single antenna selection, the complexity will be just $2 M_{R} M_{T}-M_{R}$. For more than 1 , additionally, the inner product among all the rows and uncorrelation are calculated. Only the lower triangular 
matrix without the diagonal elements of the correlation matrix is required to be calculated. The correlation matrix is as follows:

$$
\left(\begin{array}{cccc}
\alpha_{11} & \alpha_{12} & \cdots & \alpha_{1, M_{R}} \\
\vdots & \ddots & & \vdots \\
\alpha_{M_{R}, 1} & \alpha_{M_{R}, 2} & \cdots & \alpha_{M_{R}, M_{R}}
\end{array}\right)
$$

Hence, the total complexity of the proposed algorithm is $M_{T} M_{R}^{2}+M_{R} M_{T}+M_{R}^{2}-2 M_{R}$, where it is to be noted that the expression is not a function of $L_{R}$ and hence the total number of multiplications and summations is constant with respect to $L_{R}$. If $L_{R}=M_{R}$, no selection is required and hence no multiplications and summations are required. The corresponding real number flops for single antenna selection and more than one antenna selection are, respectively, $8 M_{T} M_{R}-4 M_{R}$ and $4 M_{T} M_{R}^{2}+4 M_{T} M_{R}-0.5 M_{R}^{2}-3.5 M_{R}$.

In the case of the algorithm given by Molisch et al. the number of complex-complex flops for selection of both more than and equal to 1 antenna is the same. The number of complex-complex flops is given by $M_{T} M_{R}^{2}+M_{R} M_{T}-$ $(1 / 2) M_{R}^{2}-(1 / 2) M_{R}$, where the complexity is slightly less than ours. It is to be noted that the authors of [12] did not make any provision for reduction of complexity for single antenna case. Also, there was no provision to suspend the selection process when $L_{R}=M_{R}$.

The corresponding real-real flops are $4 M_{T} M_{R}^{2}+4 M_{T} M_{R}-$ $2 M_{R}^{2}-2 M_{R}$ for single and more than one antenna selections. In the case of Gharavi-Gershman's greedy algorithm [11], for calculating B matrix $\left(L_{R}-1\right)$ times, $2 M_{T}^{2}$ complex-complex multiplications and complex-complex summations have to be calculated $\left(L_{R}-1\right)$ times. Similarly, a matrix, which consumes $2 M_{T}^{2}-M_{T}+4$ complex-complex flops for one calculation, has to be calculated $\left(L_{R}-1\right)$ times. Further, $\alpha_{j}$ needs to be calculated $\left(L_{R}-1\right)$ times. Hence, total flops corresponding to $\alpha_{j}$ is $\left(2 M_{T} M_{R}+M_{R}\right)\left(L_{R}-1\right)$. Hence, the total complexity of Gharavi-Gershman algorithm is $2 M_{R} M_{T}-M_{R}+\left(4 M_{T}^{2}-\right.$ $\left.M_{T}+4\right)\left(L_{R}-1\right)+\left(2 M_{T} M_{R}+M_{R}\right)\left(L_{R}-1\right)$.

The corresponding real-real flops are, respectively, given by $8 M_{T} M_{R}-4 M_{R}$ and $8 M_{T} M_{R}-4 M_{R}+\left(8 M_{T} M_{R}+14 M_{T}^{2}+\right.$ $\left.4 M_{T} L_{R}+2 M_{T}-4 M_{R}+2 L_{R}-2\right)\left(L_{R}-1\right)$ for single antenna and more than one antenna cases. The problem with this algorithm is the computational complexity depends on the number of, not only receive antennas and transmit antennas, but also selected antennas. Also, the dependence on the number of transmit antennas and the number of selected antennas is large in scale. This is to be expected because this algorithm depends on calculating the expression of (1) for selecting the antennas. The complex-complex flops and real-real flops of each of the three algorithms have been, respectively, tabulated as Tables 2 and 3 . follows.

It can be seen from the complexity analysis discussion as

(i) The Molisch et al. algorithm complexity depends on only the number of physically present receive antennas and the transmit antennas. The dependence on $M_{T}$ is in $O\left(M_{T}\right)$. Hence it does not vary with number of selected antennas and the complexity is less dependent on $M_{T}$.

(ii) The complexity of the proposed algorithm depends on only the number of physically present receive antennas and the transmit antennas. The dependence on $M_{T}$ is in $O\left(M_{T}\right)$. Hence, it does not vary with number of selected antennas and the complexity is less dependent on $M_{T}$. Though the number of multiplications and summations required is very slightly greater than the Molisch et al. algorithm, this very small increase in complexity can be easily disregarded on considering the improvement in performance in terms of capacity given by our algorithm over Molisch et al. algorithm.

(iii) The calculation to be done by the algorithm of Gharavi-Alkhansari and Gershman [11] depends on the number of selected antennas and the number of transmit antennas. The dependence is of $O\left(L_{R}\right)$ and $O\left(M_{T}^{2}\right)$. Hence, it is more computationally complex. The complexity is clearly a function of $M_{T}$ and $L_{R}$. This fact clearly imposes a constraint on choice of $M_{T}$ and $L_{R}$.

\section{Simulation Considerations and Results}

It is known that the capacity of a MIMO system is proportional to the $\min \left(M_{R}, M_{T}\right)$. In the case of receive-only selection, the capacity will be proportional to $\min \left(L_{R}, M_{T}\right)$. For $L_{R}$ greater than $M_{T}$, the capacity improvement will not be on linear order, rather on logarithmic order. Hence, if the antenna selection has to provide a linear increase up to $L_{R}$ equal to one less than $M_{R}$, it is necessary to keep $M_{T}=M_{R}$. The simulation carried out in [11] assumed that $M_{T}=4$ and $M_{R}=16$. Surely, this imposes a constraint on $L_{R}$. Such a constraint will impose discomfort in capacity maximisation. Hence, the simulation is carried out for high values of $M_{T}$, here in our case for $M_{T}=16 . M_{R}$ is assumed to be equal to 16.

The following plots have been obtained for the proposed Molisch et al. and Gharavi-Gershman algorithms:

(i) the outage capacity in bits/s/Hz versus $L_{R}$,

(ii) the outage capacity in bits/s/Hz versus SNR in $\mathrm{dB}$ for two different $L_{R}, L_{R}=6$ and 8 ,

(iii) the cumulative probability density versus Instantaneous capacity in bits/s/Hz for two different $L_{R}, L_{R}=$ 6 and 8 ,

(iv) the number of flops versus $L_{R}$.

Because of huge time involvement, the optimal way of selection simulation for outage capacity versus the number selected antennas plot has been limited to $M_{R}=M_{T}=8$ case. Also, the random selection plot has been obtained only for this case, because the authors felt that it is clear that random selection is the poorest in terms of capacity performance and hence it is not necessary to regard further. Antenna selection 
TABLE 2: Complexity in complex flops for the proposed and other algorithms.

\begin{tabular}{lccc}
\hline $\begin{array}{l}\text { Sl. } \\
\text { no. }\end{array}$ & The name of algorithm & The complexity in complex flops for more than 1 antenna & For single antenna \\
\hline 1 & Proposed & $M_{T} M_{R}^{2}+M_{R} M_{T}+M_{R}^{2}-2 M_{R}$ & $2 M_{R} M_{T}-M_{R}$ \\
2 & Molisch et al. [6] & $M_{T} M_{R}^{2}+M_{R} M_{T}-\frac{1}{2} M_{R}^{2}-\frac{1}{2} M_{R}$ & $\begin{array}{c}\text { Same as the } \\
\text { previous column }\end{array}$ \\
3 & Gharavi-Alkhansari and Gershman [11] & $2 M_{R} M_{T}-M_{R}+\left(4 M_{T}^{2}-M_{T}+4\right)\left(L_{R}-1\right)+\left(2 M_{T} M_{R}+M_{R}\right)\left(L_{R}-1\right)$ & $2 M_{R} M_{T}-M_{R}$ \\
\hline
\end{tabular}

TABLE 3: Complexity in real-real flops for the proposed and other algorithms.

\begin{tabular}{lccc}
\hline $\begin{array}{l}\text { Sl. } \\
\text { no. }\end{array}$ & The name of algorithm & The complexity in real-real flops for more than 1 antenna & For single antenna \\
\hline 1 & Proposed & $4 M_{T} M_{R}^{2}+4 M_{T} M_{R}-0.5 M_{R}^{2}-3.5 M_{R}$ & $8 M_{T} M_{R}-4 M_{R}$ \\
Same as the & previous column \\
2 & Molisch et al. [6] & $4 M_{T} M_{R}^{2}+4 M_{T} M_{R}-2 M_{R}^{2}-2 M_{R}$ & $8 M_{T} M_{R}-4 M_{R}$ \\
3 & Gharavi-Alkhansari and Gershman [11] & $\left(16 M_{T} M_{R}+14 M_{T}^{2}+4 M_{T} L_{R}+2 M_{T}-8 M_{R}+2 L_{R}-2\right)\left(L_{R}-1\right)$ & 8
\end{tabular}

approach of increasing capacity is applicable for slowfading and quasistatic channels. For such kind of channels, outage capacity concept is applicable rather than ergodic capacity concept.

For all the simulations, a 2000-channel average has been obtained. Simulation was carried out for two types channels. One of the two was of Rayleigh flat-fading type, where the elements of $\mathbf{H}$ are independent and identically distributed (i.i.d.) complex Gaussian of zero-mean and unit variance; that is, real and imaginary parts of $\mathbf{H}$ are of 0.5 variance It is assumed that the channel elements are uncorrelated; that is, there is no correlation at the transmit side antenna elements or receive side antenna elements. The other one type of the two channels for which simulation was carried out was a channel of some amount of correlation. It was assumed that there was no correlation at the base station end and there was correlation at the mobile station. This is based on the fact that there can be feasibility of maintaining sufficient decorrelating antenna separation at the base station, whereas it is difficult to maintain the same at the mobile station. The Kronecker model was used for modeling the channel matrix. The Kronecker model is as follows:

$$
\mathbf{H}=\mathbf{R}_{r}^{1 / 2} \mathbf{H}_{w} \mathbf{R}_{t}{ }^{1 / 2},
$$

where $\mathbf{R}_{r} \triangleq$ Receive antenna correlation matrix of $M_{R} \times M_{R}$, $\mathbf{R}_{t} \triangleq$ Transmit antenna correlation matrix of $M_{T} \times M_{T}, \mathbf{H}_{w} \triangleq$ Spatially white channel.

In our case, $\mathbf{R}_{t}$ is a unit matrix of $M_{T} \times M_{T}$ order. $\mathbf{R}_{r}$ is modelled in an exponential way as discussed in [16]. The model is as follows:

$$
\mathbf{R}_{r}=\left(\begin{array}{ccccc}
1 & r & r^{4} & \cdots & r^{\left(M_{R}-1\right)^{2}} \\
r & 1 & r & \ddots & \vdots \\
r^{4} & r & 1 & \ddots & r^{4} \\
\vdots & \ddots & \ddots & \ddots & r \\
r^{\left(M_{R}-1\right)^{2}} & \cdots & r^{4} & r & 1
\end{array}\right)
$$

where $r$ is the correlation coefficient between the adjacent antennas. It was assumed in our simulation setup that the correlation coefficient was 0.2 . In such a case, the correlation between nonadjacent antennas can be neglected and the corresponding terms in the correlation matrix can be set to be zero. Consequently, the receive correlation matrix becomes

$$
\mathbf{R}_{r}=\left(\begin{array}{ccccc}
1 & r & 0 & \cdots & 0 \\
r & 1 & r & \ddots & 0 \\
0 & r & 1 & \ddots & 0 \\
\vdots & \ddots & \ddots & \ddots & r \\
0 & \cdots & 0 & r & 1
\end{array}\right)
$$

where $r=0.2$.

It can be concluded from Figure 4 that the outage capacity performance with respect to $L_{R}$ of the proposed algorithm is superior to that of Molisch et al. algorithm. It is only slightly lower than the optimal one, and Gharavi-Gershman algorithm. The plots have been obtained for the proposed algorithm, the two other algorithms mentioned earlier, the optimal one, and random one. The norm-based algorithm is not considered, as it is known that it is applicable for only low SNR condition, though the complexity is very low. Figure 3 describes the variation of outage capacity with the number of selected antennas for $M_{T}=8, M_{R}=8$, and SNR $=20 \mathrm{~dB}$. The plots have been obtained for random and optimal selection in addition to the three algorithm-based selections. Figure 4 gives the simulation plot of outage capacity versus number of selected antennas for $M_{T}=16, M_{R}=16$, and SNR $=20 \mathrm{~dB}$. The variation of outage capacity in bits/s/ $\mathrm{Hz}$ with the number of selected antennas for $M_{T}=M_{R}=16$ is tabulated as Table 4 for comparison clarity. It can be seen in the table that the capacity of the proposed algorithm is almost equal to that of Gharavi-Gershman algorithm and clearly superior to the original Molisch et al. algorithm. Plot as shown in Figure 5 has been obtained for cumulative probability density versus the instantaneous capacity for two different $L_{R}$ values, 6 , and 8 with $M_{T}=16$ and $M_{R}=16$. It can be seen from Figure 5 that the capacity distribution of our algorithm is superior to Molisch et al. algorithm and the lowest capacity is only 


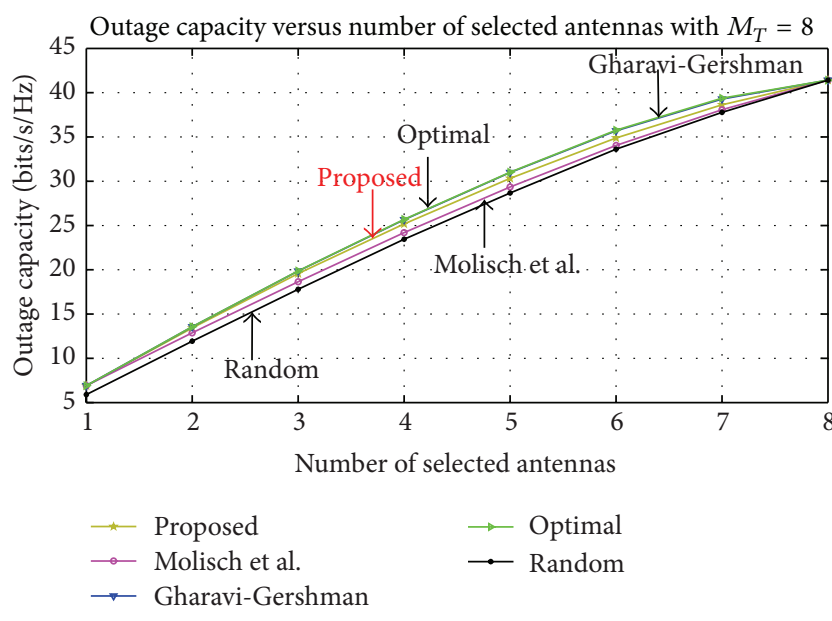

Figure 3: Plots showing the variation of outage capacity with number of selected antennas for $M_{T}=M_{R}=8$ under i.i.d. channel condition.

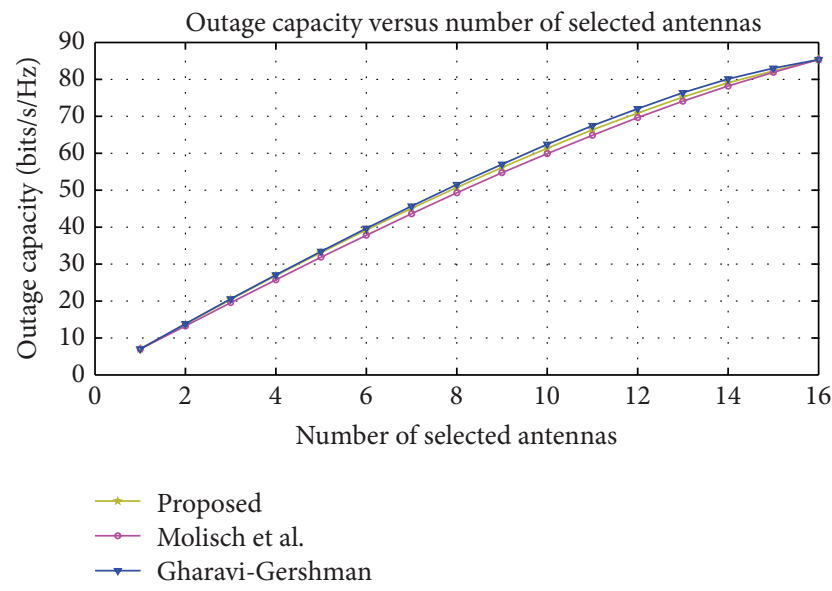

FIGURE 4: Plots showing the variation of outage capacity with number of selected antennas for $M_{T}=16$ and $M_{R}=16$ under i.i.d. channel condition.

slightly lower than that of Gharavi-Gershman. Plots as shown in Figure 6 have been obtained to see the relation between outage capacity and SNR in $\mathrm{dB}$. Under low SNR condition, the proposed algorithm and Gharavi-Gershman algorithms perform almost at the same level. However Molisch et al. algorithm suffers. This suffering is more for high SNRs.

Figure 7 shows simulated plots describing variation of outage capacity with $L_{R}$ for $M_{R}=M_{T}=16$ under correlated channel condition. It may be seen that the capacity reduces in general. It may also be noted that the capacity performance of the proposed algorithm slightly reduces. Since the proposed algorithm falls under uncorrelation concept, the correlation slightly affects the performance. Figure 8 is on variation of cumulative probability density with instantaneous capacity for $M_{R}=M_{T}=16$ and $L_{R}=6$ and 8 under correlated channel condition. Figure 9 is on variation of outage capacity with SNR in $\mathrm{dB}$ for $M_{R}=M_{T}=16$ and $L_{R}=6$ and 8 under correlated channel condition.
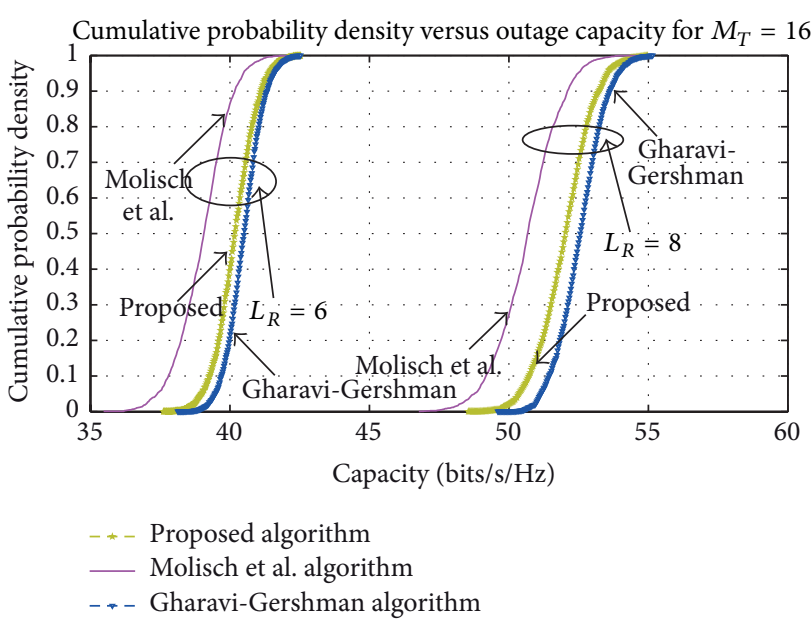

FIGURE 5: Plots showing the variation of cumulative probability density versus instantaneous capacity for $M_{T}=16$ and $M_{R}=16$ for $L_{R}=6$ and 8 under i.i.d. channel condition.

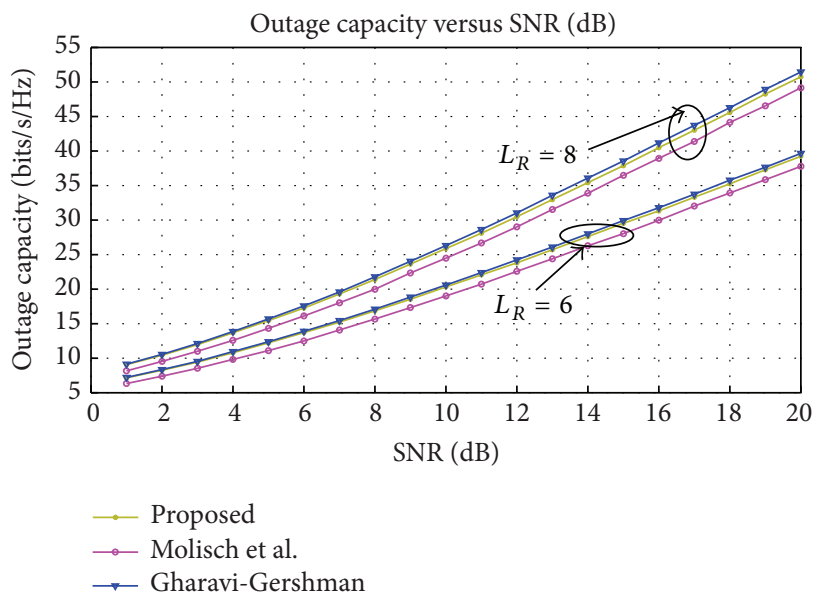

FIGURE 6: Plots showing the variation of outage capacity versus SNR in $\mathrm{dB}$ for $M_{T}=16, M_{R}=16$, and $L_{R}=6$ and 8 under i.i.d. channel condition.

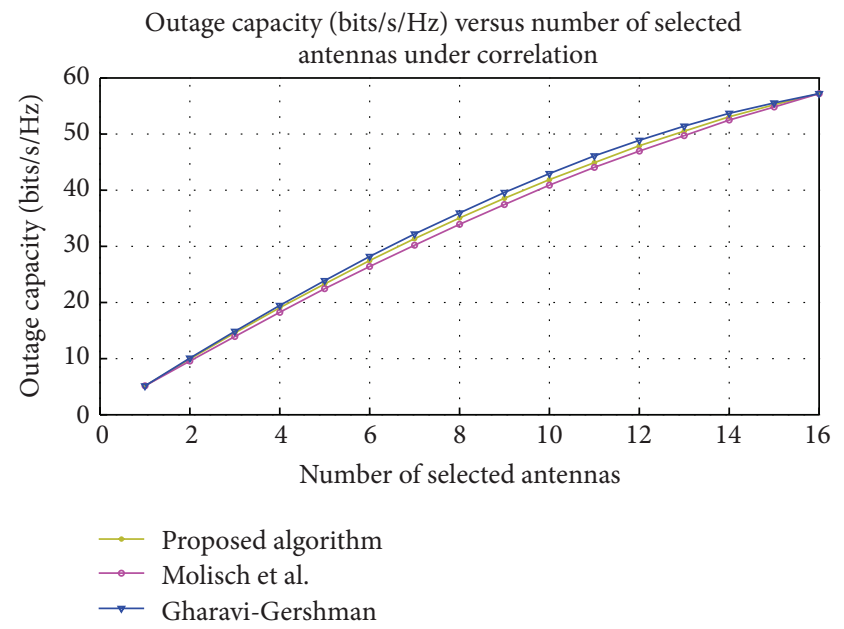

FIGURE 7: Plots showing variation of outage capacity with number of selected antennas for $M_{T}=16$ and $M_{R}=16$ under correlated condition. 
TABLE 4: Variation of outage capacity with $L_{R}$ for $M_{T}=M_{R}=16$.

\begin{tabular}{|c|c|c|c|c|c|}
\hline \multirow{2}{*}{$\begin{array}{l}\text { No. of } \\
\text { ant. }\end{array}$} & \multirow{2}{*}{$\begin{array}{c}\text { Capacity by } \\
\text { Ghar-Gersh in bps/Hz }\end{array}$} & \multirow{2}{*}{$\begin{array}{c}\text { Capacity by } \\
\text { Molisch in bps/Hz }\end{array}$} & \multirow{2}{*}{$\begin{array}{l}\text { Capacity by } \\
\text { Proposed one in bps/Hz }\end{array}$} & \multicolumn{2}{|c|}{$\begin{array}{c}\text { Increase of Ghar-Gersh real-real flops over the } \\
\text { proposed as a per cent of the proposed }\end{array}$} \\
\hline & & & & Complex-Complex & Real-Real \\
\hline 1 & 7.01 & 7.01 & 7.01 & 0.0 & 0.0 \\
\hline 2 & 13.85 & 13.25 & 13.79 & -55.5 & -56.7 \\
\hline 3 & 20.59 & 19.55 & 20.43 & -21.9 & -25.6 \\
\hline 4 & 27.09 & 25.76 & 26.88 & 11.8 & 4.7 \\
\hline 5 & 33.44 & 31.88 & 33.13 & 45.5 & 34.3 \\
\hline 6 & 39.68 & 37.79 & 39.25 & 79.1 & 63.2 \\
\hline 7 & 45.70 & 43.63 & 45.10 & 112.8 & 91.4 \\
\hline 8 & 51.54 & 49.31 & 50.75 & 146.4 & 118.9 \\
\hline 9 & 57.02 & 54.74 & 56.16 & 180.1 & 145.6 \\
\hline 10 & 62.43 & 59.90 & 61.34 & 213.7 & 171.6 \\
\hline 11 & 67.48 & 64.87 & 66.37 & 247.4 & 196.9 \\
\hline 12 & 72.07 & 69.66 & 70.87 & 281.0 & 221.5 \\
\hline 13 & 76.40 & 74.10 & 75.21 & 314.7 & 245.4 \\
\hline 14 & 80.12 & 78.22 & 79.10 & 348.3 & 268.5 \\
\hline 15 & 83.03 & 81.90 & 82.28 & 382.0 & 290.9 \\
\hline 16 & 85.36 & 85.36 & 85.36 & - & - \\
\hline
\end{tabular}

TABLE 5: Complexity break-up of Gharavi-Alkhansari and Gershman algorithm [11].

\begin{tabular}{|c|c|c|c|}
\hline Sl. no. & Algorithm statements & Complex flops required & Real flops required \\
\hline 1 & $S_{R}=\left\{1,2,3, \ldots, M_{R}\right\}$ & & \\
\hline 2 & $\mathbf{B}=\mathbf{I}_{M_{T}}$ & & \\
\hline 3 & for $j=1$ to $M_{R}$ & & \\
\hline 4 & $\alpha_{j}=\left\|\mathbf{H}_{j,:}\right\|^{2}$ & $2 M_{R} M_{T}-M_{R}$ & $8 M_{R} M_{T}-4 M_{R}$ \\
\hline 5 & end & & \\
\hline 6 & for $n=1$ to $L_{R}$ & & \\
\hline 7 & $J=\arg \max \alpha_{j}$ & & \\
\hline 8 & $j \in S_{R}$ & & \\
\hline 9 & $S_{R}=S_{R}-J$ & & \\
\hline 10 & If $n<L_{R}$ & & \\
\hline 11 & $\mathbf{a}=\frac{1}{\sqrt{L_{T} / \rho+\alpha_{J}}} \mathbf{B} \mathbf{H}_{J,:}^{H}$ & $\left(2 M_{T}^{2}-M_{T}+4\right)\left(L_{R}-1\right)$ & $\left(6 M_{T}^{2}+2 M_{T}\right)\left(L_{R}-1\right)$ \\
\hline 12 & $\mathbf{B}=\mathbf{B}-\mathbf{a a}^{H}$ & $\left(2 M_{T}^{2}\right)\left(L_{R}-1\right)$ & $\left(8 M_{T}^{2}-2\right)\left(L_{R}-1\right)$ \\
\hline 13 & for all $j \in S_{R}$ & \multirow{5}{*}{$\left(2 M_{T} M_{R}+M_{R}\right)\left(L_{R}-1\right)$} & \multirow{5}{*}{$\left(8 M_{T}-4\right)\left(M_{R}-\frac{L_{R}}{2}\right)\left(L_{R}-1\right)$} \\
\hline 14 & $\alpha_{j}=\alpha_{j}-\left|\mathbf{a}^{H} \mathbf{H}_{j,:}^{H}\right|^{2}$ & & \\
\hline 15 & end & & \\
\hline 16 & end & & \\
\hline 17 & end & & \\
\hline 18 & $\mathfrak{E}_{R}=\left\{1,2,3, \ldots, M_{R}\right\}-S_{R}$ & & \\
\hline
\end{tabular}

Figure 10 is on the number of real-real flops versus the number of selected antennas. As seen in the figure, the number of flops demanded by the proposed algorithm is only very slightly greater than the Molisch et al. algorithm. However, the performance gain is of a significant amount as seen in Figure 3 to Figure 9. As seen in Figure 10, GharaviGershman algorithm complexity increases almost linearly with $L_{R}$. Columns 5 and 6 of Table 4 show complex-complex and real-real flop increase with $L_{R}$ of Gharavi-Gershman as a percent complexity of the proposed algorithm. Practically, real-real flops matter rather than complex-complex flops. Clearly, our algorithm performs well both in performance and complexity. Our algorithm will be a good choice for systems, which need to have liberty on $L_{R}$ up to $M_{R}-1$ 
TABLE 6: Complexity break-up of Molisch et al. algorithm [6].

\begin{tabular}{|c|c|c|c|}
\hline Sl. no. & Algorithm statements & Complex flops required & Real flops required \\
\hline 1 & $S_{R}=\left\{1,2,3, \ldots, M_{R}\right\}$ & & \\
\hline 2 & for $j=1$ to $M_{R}$ & \multirow{3}{*}{$2 M_{R} M_{T}-M_{R}$} & \multirow{3}{*}{$8 M_{R} M_{T}-4 M_{R}$} \\
\hline 3 & $\gamma_{j}^{2}=\left\|\mathbf{H}_{j,:}\right\|^{2}$ & & \\
\hline 4 & end & & \\
\hline 5 & for $p=1$ to $M_{R}$ & \multirow{13}{*}{$\frac{\left(2 M_{T}-1\right)\left(M_{R}^{2}-M_{R}\right)}{2}$} & \multirow{13}{*}{$\left(4 M_{T}-2\right)\left(M_{R}^{2}-M_{R}\right)$} \\
\hline 6 & for $q=1$ to $M_{R}$ & & \\
\hline 7 & if $p>q$ & & \\
\hline 8 & $\alpha_{p, q}=\mathbf{H}_{p,:} \quad \mathbf{H}_{q,:}^{H}$ & & \\
\hline 9 & end & & \\
\hline 10 & end & & \\
\hline 11 & end & & \\
\hline 12 & for $n=1$ to $M_{R}-L_{R}$ & & \\
\hline 13 & $P, Q=\underset{p, q \in S_{R}}{\arg \max } \alpha_{p, q}$ & & \\
\hline 14 & if $\left\|\mathbf{H}_{P,:}\right\|^{2}>\left\|\mathbf{H}_{\mathrm{Q}:}\right\|^{2}$ & & \\
\hline 15 & $S_{R}=S_{R}-Q$ & & \\
\hline 16 & $\alpha_{\mathrm{Q},:}=0$ & & \\
\hline 17 & else & & \\
\hline 18 & $S_{R}=S_{R}-P$ & & \\
\hline 19 & $\alpha_{P,:}=0$ & & \\
\hline 20 & end & & \\
\hline 21 & end & & \\
\hline 26 & $\mathfrak{E}_{R}=S_{R}$ & & \\
\hline
\end{tabular}

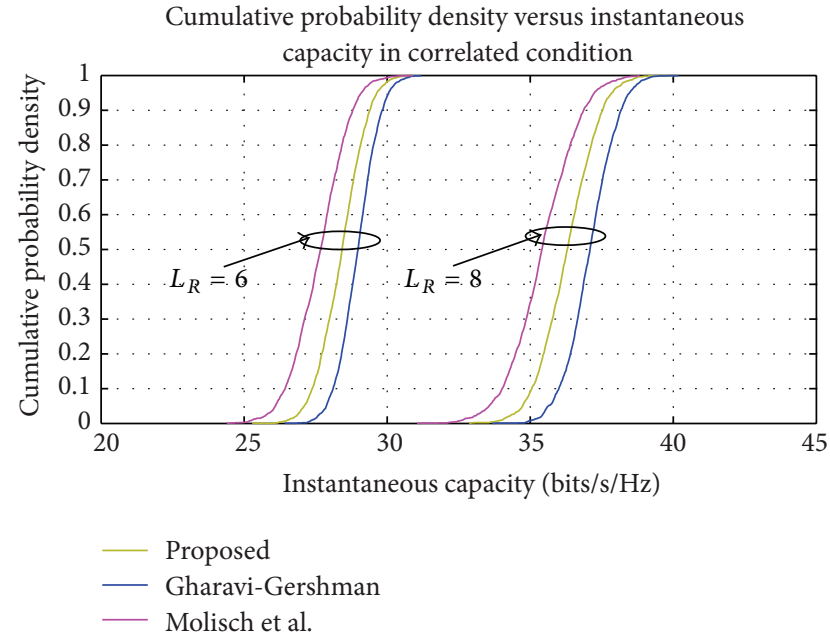

FIGURE 8: Plots showing the variation of cumulative probability density versus instantaneous capacity for $M_{T}=16, M_{R}=16$ and $L_{R}=6$ and 8 under correlated.

with linear increase in capacity with regard to increase in $L_{R}$.

\section{Conclusion}

In this paper, a low complexity algorithm was formulated and the detailed steps of the algorithm were stated with the complexity involvement. The complexities of other existing

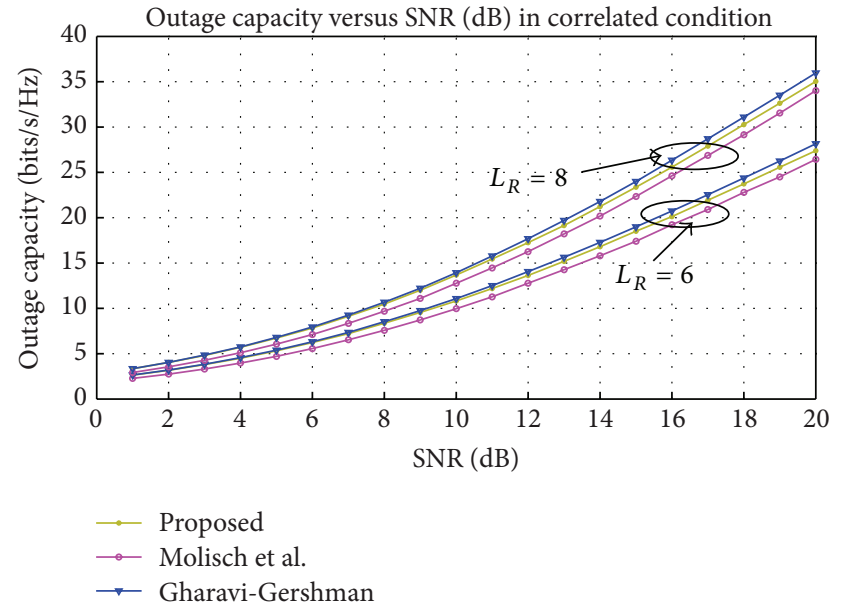

FIGURE 9: Plots showing the variation of outage capacity versus SNR in $\mathrm{dB}$ for, $M_{T}=16, M_{R}=16$ and $L_{R}=6$ and 8 under correlated condition.

algorithms were calculated. For all the three algorithms, complex-complex flops and real-real flops were calculated and a comparison was done. The performances of all the algorithms were seen in i.i.d. and correlated channel conditions. Our algorithm is balanced in terms of performance and complexity. Our algorithm will be a good choice for systems which need to have liberty on $L_{R}$ up to $M_{R}-1$ with linear increase in capacity with regard to increase in $L_{R}$. 


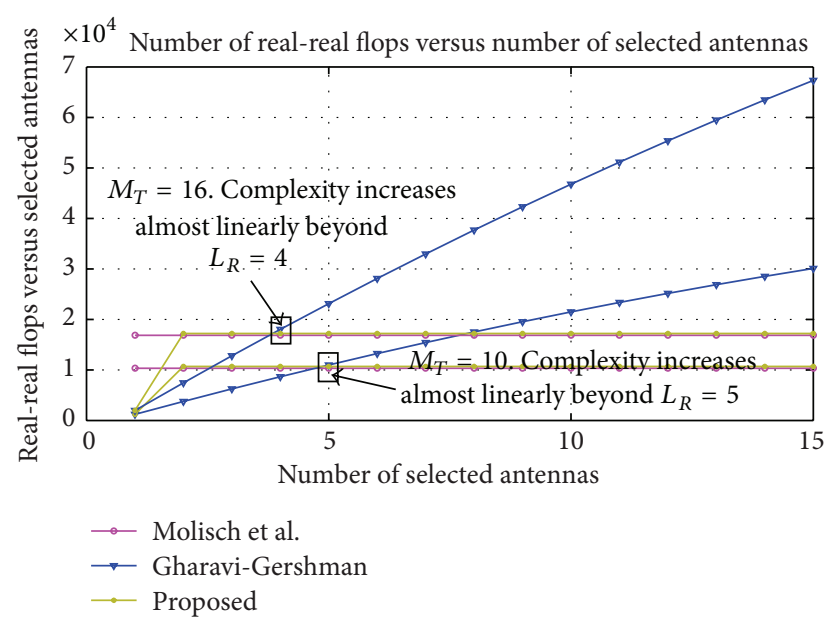

FigUre 10: Plots showing the variation of real-real flops as a function of the number of selected antennas for $M_{T}=16$ and 10 and $M_{R}=16$.

\section{Acknowledgments}

The authors are very grateful to the reviewers for the useful and constructive comments they have given after going through the paper thoroughly. The comments have further motivated the authors towards research and furthered their research aptitude.

\section{References}

[1] E. Telatar, "Capacity of multi-antenna Gaussian channels," European Transactions on Telecommunications, vol. 10, no. 6, pp. 585-595, 1999.

[2] J. H. Winter and M. Z. Win, "Hybrid-selection/opimum combining," in Proceedings of the IEEE Vehicular Technology Conference (VTC '01), pp. 113-117, 2001.

[3] D. A. Gore, R. U. Nabar, and A. Paulraj, "Selecting an optimal set of transmit antennas for a low rank matrix channel," in Proceedings of the IEEE Interntional Conference on Acoustics, Speech, and Signal Processing (ICASSP '00), pp. 2785-2788, June 2000.

[4] T. R. Ramya and S. Bhashyam, "Rate adaptation in MIMO antenna selection system with imperfect CSIT," in Proceedings of the 2nd International Conference on Communication Systems and NETworks (COMSNETS '10), January 2010.

[5] R. W. Heath Jr., S. Sandhu, and A. Paulraj, "Antenna selection for spatial multiplexing systems with linear receivers," IEEE Communications Letters, vol. 5, no. 4, pp. 142-144, 2001.

[6] A. F. Molisch, M. Z. Win, and J. H. Winters, "Capacity of MIMO systems with antenna selection," in Proceedings of the International Conference on Communications (ICC'01), pp. 570574, June 2000.

[7] D. A. Gore, R. W. Heath Jr., and A. J. Paulraj, "Transmit selection in spatial multiplexing systems," IEEE Communications Letters, vol. 6, no. 11, pp. 491-493, 2002.

[8] D. Gore and A. Paulraj, "Space-time block coding with optimal antenna selection," in Proceedings of the IEEE Interntional Conference on Acoustics, Speech, and Signal Processing (ICASSP '01), pp. 2441-2444, May 2001.
[9] S. Sanayei and A. Nosratinia, "Antenna selection in MIMO systems," IEEE Communications Magazine, vol. 42, no. 10, pp. 68-73, 2004.

[10] A. Gorokhov, "Antenna selection algorithms for MEA transmission systems," in Proceedings of the IEEE International Conference on Acoustic, Speech, and Signal Processing (ICASSP '02), pp. 2857-2860, May 2002.

[11] M. Gharavi-Alkhansari and A. B. Gershman, "Fast antenna subset selection in MIMO systems," IEEE Transactions on Signal Processing, vol. 52, no. 2, pp. 339-347, 2004.

[12] A. F. Molisch, M. Z. Win, Y.-S. Choi, and J. H. Winters, "Capacity of MIMO systems with antenna selection," IEEE Transactions on Wireless Communications, vol. 4, no. 4, pp. 1759-1771, 2005.

[13] Y. Haifen and L. Guangjun, "Novel antenna selection algorithm based on Tanimoto similarity," Journal of Systems Engineering and Electronics, vol. 19, no. 3, pp. 624-627, 2008.

[14] A. Gorokhov, M. Collados, D. Gore, and A. Paulraj, "Transmit/receive MIMO antenna subset selection," in Proceedings of the IEEE International Conference on Acoustics, Speech, and Signal Processing (ICASSP '04), pp. 13-16, May 2004.

[15] C.-E. Chen, "A computationally efficient near-optimal algorithm for capacity- maximization based joint transmit and receive antenna selection," IEEE Communications Letters, vol. 14, no. 5, pp. 402-404, 2010.

[16] G. Tsoulos, MIMO System Technology for Wireless Communications, CRC Press, Taylor and Francis Group, 2006. 

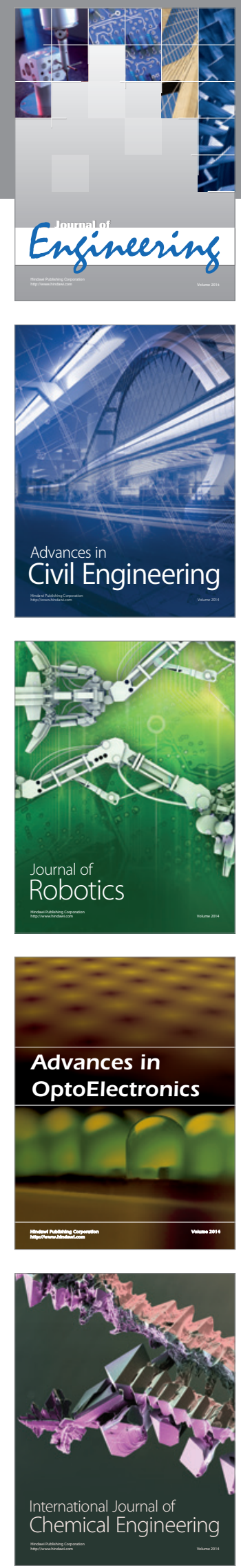

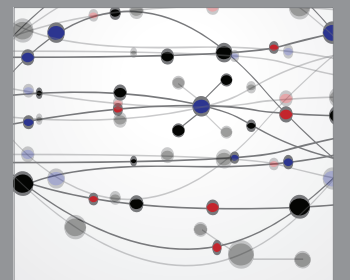

The Scientific World Journal
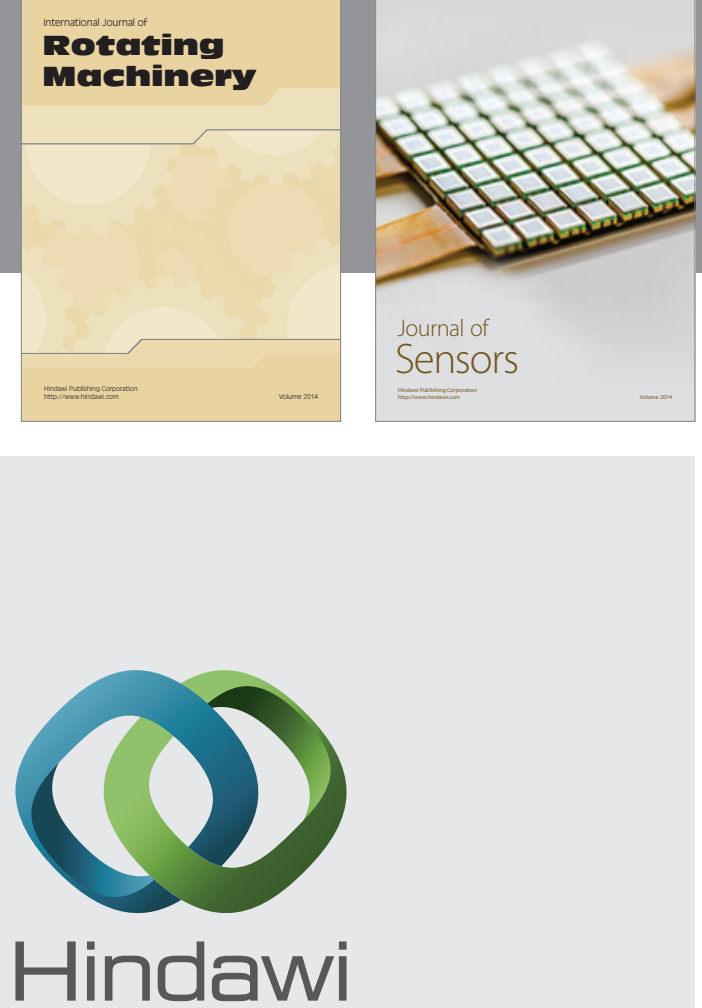

Submit your manuscripts at http://www.hindawi.com
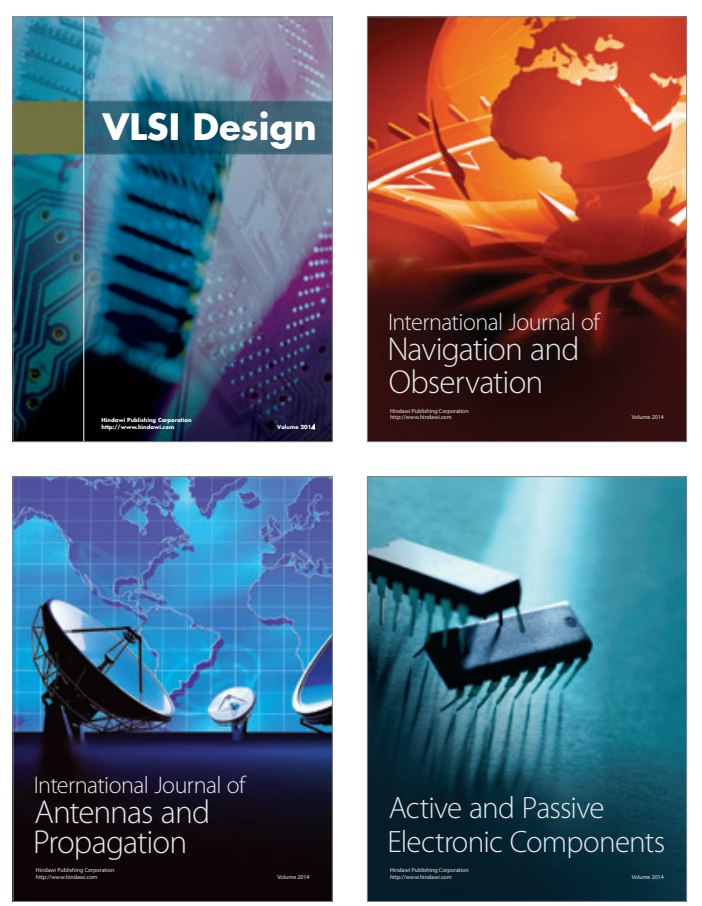
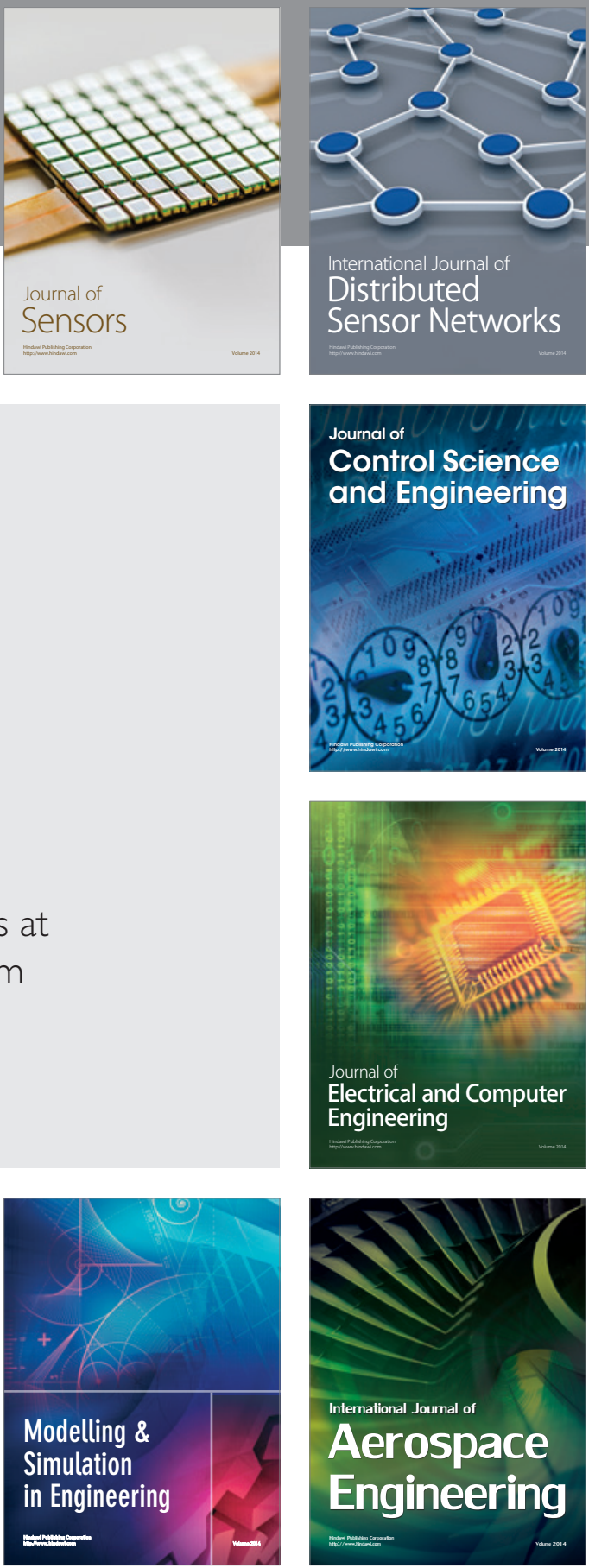

Journal of

Control Science

and Engineering
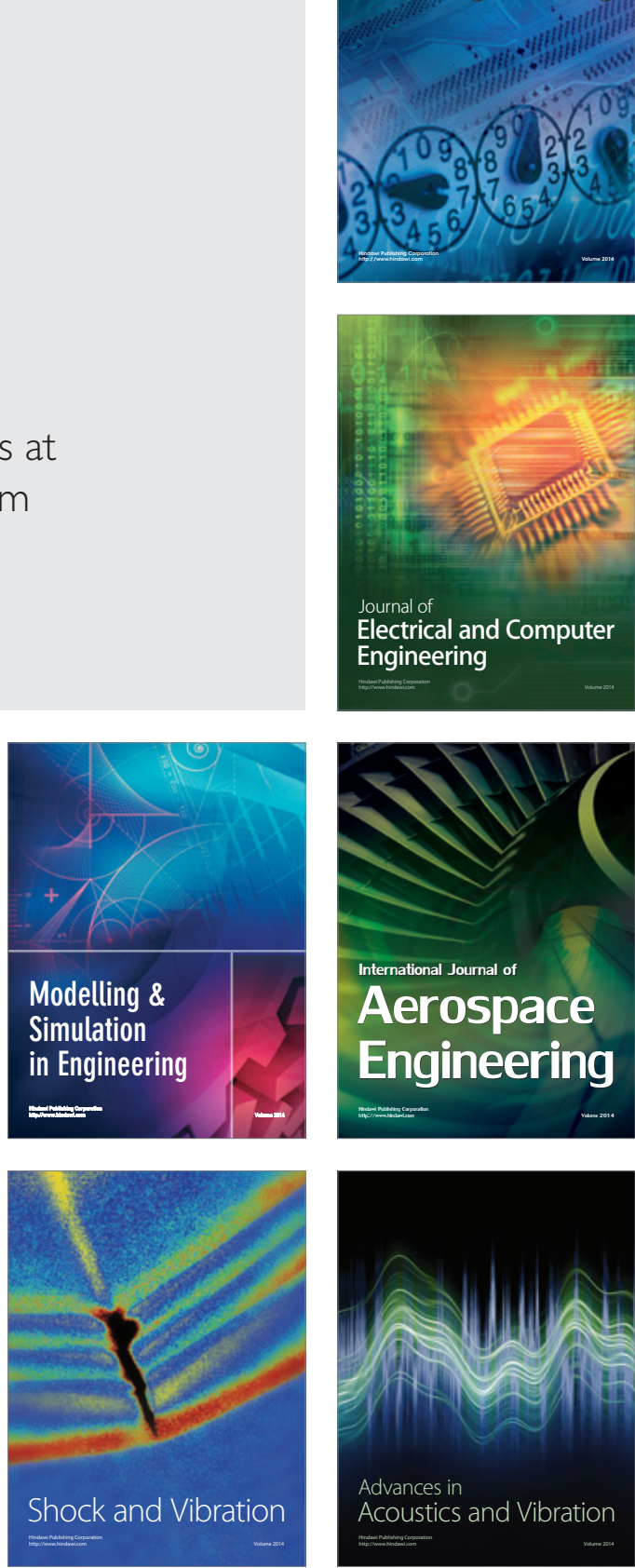